\title{
Endothelial function in patients with critical and non-critical limb ischemia undergoing endovascular treatment
}

\author{
Paweł Kaczmarczyk', Marzena Frołow ${ }^{1,4}$, Rafał Januszek2,3, Andrzej Belowski', Iwona Gregorczyk-Maga ${ }^{6}$, \\ Stefan Chlopicki ${ }^{4,5}$, Paweł Maga'
}

'Department of Angiology, Jagiellonian University Medical College, Kraków, Poland

${ }^{2} 2^{\text {nd }}$ Department of Cardiology and Cardiovascular Interventions, University Hospital, Kraków, Poland

${ }^{3}$ Department of Clinical Rehabilitation, University of Physical Education, Kraków, Poland

${ }^{4}$ Jagiellonian Centre for Experimental Therapeutics, Jagiellonian University, Kraków, Poland

${ }^{5}$ Chair of Pharmacology, Jagiellonian University Medical College, Kraków, Poland

'Institute of Dentistry, Jagiellonian University Medical College, Kraków, Poland

\author{
Correspondence to: \\ Paweł Maga, MD, PhD, \\ Department of Angiology, \\ Jagiellonian University \\ Medical College, \\ Jakubowskiego 2, 30-688 \\ Kraków, Poland, \\ phone: +48 124003262 \\ e-mail: \\ maga.pawel@gmail.com \\ Copyright by the \\ Author(s), 2021 \\ Kardiol Pol. 2021; \\ 79 (7-8): 804-812; \\ DOl: 10.33963/KP.a2021.0010 \\ Received: \\ March 23, 2021 \\ Revision accepted: \\ May 17, 2021 \\ Published online: \\ May 18, 2021
}

\begin{abstract}
A B S T R A C T
Background: Critical limb ischemia (CLI) is the most advanced stage of peripheral arterial disease. CLI patients, compared to non-CLI, achieve worse treatment outcomes and generate higher costs.

Aims: The aim of the study was to compare endothelial function and clinical outcomes in CLI and non-CLI patients after percutaneous transluminal angioplasty (PTA).

Methods: In this prospective, follow-up study, $30 \mathrm{CLI}$ patients and 40 non-CLI patients underwent PTA. Endothelial function was assessed based on flow mediated dilatation (FMD), reactive-hyperemia index $(\mathrm{RHI})$, while the ankle-brachial index, toe-brachial index and the Rutherford scale were used for peripheral artery disease progression evaluation. The results were assessed before PTA, as well as 1, 3, 6 and 12 months after the procedure.
\end{abstract}

Results: There were no differences at the baseline regarding to endothelial function between both groups. Neither FMD nor RHI changed after PTA in any of the groups, although there was a difference in median $\mathrm{RHI}$ value between $\mathrm{CLI}$ and non-CLI patients regarding the $1^{\text {st }}$ and $6^{\text {th }}$ month of the follow-up $(\mathrm{RHI} 6-\mathrm{RHI} 1=0.08$ in $\mathrm{CLI}$ and -0.15 in non-CLI; $P=0.01)$. The larger baseline intima-media thickness (IMT) in the CLI group allowed to predict a greater number of re-intervention $(P=0.01)$ and major adverse event rates $(P=0.03)$. CLI patients presented larger decrease in the Rutherford scale compared to non-CLI $(P<0.001)$.

Conclusions: Baseline IMT was predictive for re-interventions and major adverse event rates. Although neither of groups exhibited significant changes in endothelial function, we proved differences between them regarding to changes in $\mathrm{RHI}$.

Key words: critical limb ischemia, endothelium, endovascular treatment, peripheral artery disease, revascularization

Kardiol Pol 2021; 79, 7-8: 804-812

\section{INTRODUCTION}

Peripheral artery disease (PAD) is characterized by ischemia in the lower limbs due to narrowing of the arteries because of atherosclerotic plaque accumulation [1]. Critical limb ischemia (CLI) is the most advanced stage of PAD associated with macro- and microcirculatory, as well as rheological disorders. The Inter-Society Consensus for the Management of PAD estimates that $25 \%$ of patients diagnosed with CLI will die within 1 year and an additional $30 \%$ will undergo limb amputation [2]. CLI patients typically present a spec- trum of symptoms including pain during rest, non-healing ulcers, and tissue necrosis with gangrene [3]. Limb revascularization is considered the first line of treatment for CLI because it significantly reduces the rate of limb amputation and mortality $[4,5]$. Comorbidities are more frequent in CLI patients, who require faster treatment access and more advanced procedural strategies. These facts make them more vulnerable to procedural complications [6-8]. Despite clinical differences between patients with CLI and those with limb claudication (non-CLI), they both exhibit 
WHAT'S NEW?

In the majority of clinical studies related to peripheral arterial disease, critical limb ischemia (CLI) patients were analyzed together with non-critical limb ischemia (non-CLI) patients. Thus, it is unknown whether endothelial function after endovascular procedures changes in a similar way among CLI and non-CLI patients. To our knowledge, we are the first to investigate if there are any significant differences regarding endothelial function (flow mediated dilatation), intima-media thickness, or clinical outcomes separately in these 2 groups. It is suggested in the present study that CLI patients had better clinical response to revascularization than non-CLI patients (larger drop in the Rutherford scale, better increase in toe-brachial index [TBI]), however, there was no improvement in endothelial function after revascularization in either group.

endothelial dysfunction, the hallmark of atherosclerosis pathogenesis. In fact, endothelium-dependent vasodilation is impaired in coronary artery disease patients $[9,10]$. Interventions proven to reduce cardiovascular risk also reverse endothelial dysfunction and failure of the endothelium to respond to therapy [11]. Endothelial dysfunction is also present in PAD patients. The loss of nitric oxide-dependent regulation regarding flow in the lower limbs may worsen the vasoconstrictor effects of catecholamines and impair flow mediated dilatation (FMD), which, in consequence, may worsen stenosis severity while increasing resistance to blood flow during exercise [12].

Understanding the heterogeneity of these two groups, we should expect many differences concerning clinical aspects between patients with claudication and critical limb ischemia, especially after revascularization. Nevertheless, in the majority of clinical studies related to PAD, CLI patients were analyzed together with non-CLI patients. It is not known whether endothelial function after endovascular procedures changes in a similar way in CLI and non-CLI patients.

Therefore, here, we compared endothelial function and clinical outcomes in CLI and non-CLI patients after percutaneous transluminal angioplasty (PTA) during a 12-month follow-up period.

\section{METHODS}

\section{Study design}

The study was conducted as a prospective, single-center follow-up evaluation. Patients with critical limb ischemia as well as those with stable PAD (Rutherford class from 2 to 3 ), due to iliac, femoropopliteal, or below-the-knee disease, were eligible for the study. Exclusion criteria were history of end-stage kidney disease, age above 85 , and pain related to limb ischemia not allowing to maintain a horizontal position. Patients with incompressible tibial arteries were not eligible for the study.

All subjects provided their written and informed consent before beginning the trial. The study complies with the 1964 Declaration of Helsinki and was approved by the local ethical committee.

\section{Endovascular procedures}

Endovascular treatment was performed in a routine manner. A 4 French $(F)$ to $6 F$ sheath was introduced into the artery and diagnostic angiography was performed. Each individual received 5000 International Units of unfractionated heparin that was injected intra-arterially. The affected artery was treated using over-the-wire balloon catheters, and wherever necessary, nitinol self-expanding or expandable cobalt-chromium balloon stents. Post-interventional therapy lasted 4 weeks and consisted of both aspirin (75 mg/d) and clopidogrel (75 mg/d). Highdose statins (atorvastatin $40 \mathrm{mg}$ to $80 \mathrm{mg}$ or rosuvastatin $20 \mathrm{mg}$ to $40 \mathrm{mg}$ ) were initiated at baseline assessment for all patients and were maintained throughout the study, although compliance was not monitored. Follow-up visits were conducted 1,6 , and 12 months after the intervention. Successful angioplasty was defined by a final angiogram with residual stenosis of $30 \%$ or less, and post-interventional ankle-brachial index (ABI) improvement of at least 0.1 .

\section{Assessment of FMD}

The study was performed on the basis of current FMD assessment guidelines [13]. Patients were examined in fasting state, in supine position. They were asked to refrain from smoking, as well as alcohol and caffeinated beverage consumption in the 12 hours preceding the trial. All vasoactive drugs were discontinued 24 hours before the procedure. FMD was performed in a quiet, temperature-controlled room $\left(21^{\circ} \mathrm{C}\right.$ to $\left.24^{\circ} \mathrm{C}\right)$. Measurements were performed on the dominant forearm. A B-mode brachial artery image was obtained using a 14-MHz linear-array transducer with the Siemens Accuson 2000 ultrasound. The longitudinal segment above the antecubital fossa was used to measure brachial artery diameter.

At baseline, a 10-second clip was recorded to measure the baseline artery diameter. Then, a blood pressure cuff was inflated on the forearm to $40 \mathrm{~mm} \mathrm{Hg}$ above systolic pressure for 5 minutes. Immediately after cuff deflation, maximal blood velocity was measured for 15 seconds to evaluate arterial flow. Next, a 120-second video clip of the brachial artery in B-mode was recorded to calculate the post-occlusion brachial artery diameter. Continuous analysis of changes in the artery diameter were performed during post-processing at the authors'laboratory by means of a wall-tracking computer system developed by Zieliński et al. [14]. The maximal diameter (maximal FMD — \% FMD) was obtained using a semi-automatic technique, operating on the basis of the two regions of interest (ROI), indicating 
the anterior and posterior artery wall marked by the operator on the first frame of the ultrasound clip. The algorithm for tracking the borders of the arterial walls was based on the active contour method.

\section{Assessment of the Reactive Hyperemia Index (RHI)} Digital pulse amplitude was measured simultaneously with FMD in a fasting state and supine position using a portable appliance testing (PAT) device comprising a pneumatic plethysmograph measuring digital pulse volume changes (Endo-PAT2000, Itamar Medical, Caesarea, Israel) [15]. The digital pulse amplitude was acquired continually during the examination and digitally recorded to a laptop. Data were analyzed by a computerized algorithm (Itamar Medical), which automatically and operator-independently calculates Assessment of RHI.

\section{Pulse Wave Analysis (PWA)}

Arterial pulse waveform assessment of arterial stiffness was performed non-invasively with the commercially available SphygmoCor system (AtCor Medical). Peripheral pressure waveforms were recorded from the radial artery at the wrist, using applanation tonometry with a high-fidelity micromanometer. After 20 sequential waveforms had been acquired, a validated generalized transfer function was used to generate the corresponding central aortic pressure waveform. AP was defined as the maximal systolic pressure minus pressure at the inflection point. We measured pulse pressure (PP), augmentation index (Al), central augmentation index (CAI), ejection duration (ED), subendocardial viability ration (SEVR), central augmentation pressure (CAP), CAP normalized for the heart rate of 75 beats per minute (CAP-HR75), stiffness index (SI), and reflection index (RI).

\section{Assessment of lower limb ischemia}

$A B I$ was calculated with the patient in supine position. The highest systolic pressure of the anterior or posterior tibial artery was measured in each limb and then divided by the highest brachial artery pressure. The mean $A B I$ value for the two legs was included in statistical analysis. Toe-brachial index [TBI] was also calculated with the patient in supine position. The systolic pressure on the big toe was obtained for each limb using a photoplethysmograph (Nicolet VasoGuard; VIASYS Healthcare, Madison, WI, USA), and was divided by the highest brachial artery pressure. Painfree walking distance and maximal walking distance were measured in each patient before PTA and at every follow-up visit using a treadmill exercise test.

\section{Measurements of intima-media thickness (IMT)}

To measure carotid IMT, ultrasonography of the common carotid artery, carotid bifurcation and internal carotid artery of the left and right carotid arteries was performed with a $7.5 \mathrm{MHz}$ linear-array transducer (Siemens, Erlangen, Germany). On a longitudinal, two-dimensional ultrasound image of the carotid artery, the anterior and posterior walls of the carotid artery are displayed as two bright white lines separated by hypoechogenic space. The distance between the leading edge of the first, far wall bright line (lumen-intima interface) and the leading edge of the second bright line (media-adventitia interface) indicates the IMT.

\section{Follow-up of patients}

All of the treated patients were followed at the University Hospital Angiology Outpatient Clinic for a period of 12 months following the procedure. Endothelial function was assessed before as well as 1, 3, and 6 months after PTA based on FMD and the reactive-hyperemia index (RHI), while arterial stiffness was evaluated using arterial PWA. Clinical status was evaluated before as well as $1,3,6$, and 12 months following PTA using the ABI or TBI. The carotid artery was examined before and 1, 3, and 6 months after PTA via IMT measurement. The outcomes were analyzed separately. The primary outcome measures of the study were changes in endothelial function (FMD, RHI, arterial PWA), clinical status (ABI, TBI), and IMT after PTA. The secondary outcome measures were freedom from major adverse limb events (MALE; including occurrence of death, stroke, myocardial infarction, major amputation, and/or re-intervention). Secondary study endpoints were assessed as composite effect (MALE) and solely, all the outcomes separately.

\section{Statistical analysis}

Categorical variables are presented as numbers and percentages. Continuous variables are expressed as mean (standard deviation, [SD]) or median (lower quartile [Q1] - upper quartile [Q3]), where applicable. Normality was assessed via the Shapiro-Wilk test. Equality of variance was evaluated using the Levene's test. Differences between the two groups were compared using the Student's or Welch's t-test, depending on the equality of variances for normally distributed variables. The Mann-Whitney $U$ test was applied for non-normally distributed continuous variables. Categorical variables were compared with the Pearson's chi-squared or Fisher's exact tests if $20 \%$ of cells had an expected count of less than 5 (Monte Carlo simulation for Fisher's test using tables of higher dimensions than $2 \times 2$ ). The Wilcoxon matched pair test was used for the comparison of two dependent samples. Friedman's non-parametric ANOVA was used to compare repeated measures of more than two groups and between the groups with more than two measurements. Re-intervention rates and MALE rates in the $\mathrm{CLI}$ and non-CLI groups were compared using the log-rank test. Kaplan-Meier estimate curves were generated for MALEs and re-interventions according to the CLI status. MALEs was defined as a composite clinical endpoint including: occurrence of death, stroke, myocardial infarction, major amputation, and/or re-intervention. For comparison of relative changes in endothelial and clinical parameters between measurements at given time periods after PTA in the $\mathrm{CLI}$ and non-CLI groups, the Benjamini-Hochberg 
Table 1. Baseline patient characteristics

\begin{tabular}{|c|c|c|c|}
\hline \multirow[t]{2}{*}{ Variables } & \multicolumn{2}{|c|}{ Number of individuals, $\mathbf{n}(\%)$} & \multirow[t]{2}{*}{$P$-value } \\
\hline & CLI $(n=30)$ & Non-CLI $(n=40)$ & \\
\hline Age, years & $65.6(7.5)$ & $67.0(7.0)$ & 0.57 \\
\hline Gender, males & $24(80)$ & $31(77.5)$ & 0.8 \\
\hline Smoking, pack years & $\begin{array}{c}24(82.7) \\
30(10.4-37.5)\end{array}$ & $\begin{array}{c}38(95) \\
30(17.5-40)\end{array}$ & $\begin{array}{l}0.05 \\
0.49\end{array}$ \\
\hline Hypertension & $18(60)$ & $32(80)$ & 0.06 \\
\hline Dyslipidemia & $15(50)$ & $19(47.5)$ & 0.83 \\
\hline Diabetes mellitus, years of treatment & $\begin{array}{l}11(36.7) \\
16.3(8.4)\end{array}$ & $\begin{array}{c}16(40) \\
15.4(7.8)\end{array}$ & $\begin{array}{l}0.77 \\
0.79\end{array}$ \\
\hline Coronary artery disease & $11(36.7)$ & $15(37.5)$ & 0.94 \\
\hline Myocardial infarction & $3(10)$ & $7(17.5)$ & 0.37 \\
\hline Kidney failure (GFR $<60 \mathrm{ml} / \mathrm{min} / 1.73 \mathrm{~m}^{2}$ ) & $3(10)$ & $1(2.5)$ & 0.18 \\
\hline Cerebral stroke & $2(6.7)$ & $4(10)$ & 0.62 \\
\hline \multicolumn{4}{|l|}{ Pharmacotherapy before intervention } \\
\hline Statins & $4(13.3)$ & $2(5)$ & 0.21 \\
\hline Acetylsalicylic acid & $5(16.7)$ & $5(12.5)$ & 0.62 \\
\hline Insulin & $3(10)$ & $0(0)$ & 0.04 \\
\hline ACEIs/ARBs & $1(3.3)$ & $2(5)$ & 0.73 \\
\hline Ca-blockers & $2(6.67)$ & $3(7.5)$ & 0.89 \\
\hline$\beta$-blockers & $1(3.3)$ & $1(2.5)$ & 0.83 \\
\hline
\end{tabular}

Data are expressed as mean (SD), median (IQR) and numbers (percentages).

Abbreviations: ACEls, angiotensin converting enzyme inhibitor; ARBs, angiotensin receptor blockers; Ca-blockers, calcium channel blockers; CLI, critical limb ischemia; GFR, glomerular filtration rate

procedure was used to adjust the $P$-value [16]. Spearman correlation coefficient was calculated for comparison between selected indices. A $P$-value of $<0.05$ was considered statistically significant. STATISTICA for Windows Release 10 (Statsoft Inc., 2011) was used for data analysis.

\section{RESULTS}

\section{Patient characteristics}

Clinical characteristics of the patients included in the present study are shown in Table 1. The mean age of the CLI patients was 65.6 (7.5) years, while for non-CLI patients this totaled 67 (7) years. There were no differences in age between these two groups.

There were also no differences in pharmacological treatment or family medical history between the groups (Table 1). At the time of admission, less than $20 \%$ of patients were on statins, anti-platelet, or angiotensin converting enzyme inhibitor/ angiotensin receptor blockers-based therapy. After PTA, all patients were prescribed treatment based on aspirin with clopidogrel and high-doses of statins. Kidney failure was defined when glomerular filtration rate was less than $60 \mathrm{ml} / \mathrm{min} / 1.73 \mathrm{~m}^{2}$. Blood pressure higher than $140 / 90 \mathrm{~mm} \mathrm{Hg}$ was defined as abnormal. Since we did not measure in this study serum glucose and LDL levels, we had to accept diagnoses of diabetes and hypercholesterolemia from patients' medical history.

Considering the observational period, the mean length of the follow-up was 406.1 (209.7) days in the CLI group and 471.7 (179.2) days in the non-CLI group $(P=0.17)$. Then, the follow-up period in both groups was limited to 12 months in order to objectify the comparative analysis of follow-up results. During the 12-month follow-up, there were no amputations in the non-CLI group, but 2 amputations in the CLI group (6.7\%). There were $9(30 \%)$ re-interventions in the CLI group and $4(10 \%)$ in the non-CLI group ( $P=0.03)$. During the follow-up period, 1 patient $(2.5 \%)$ from the non-CLI group died, while no cases of death were noted in the CLI group during that time. Summarizing, there were 11 (36.7\%) MALEs in the CLI group and 5 (12.5\%) in the non-CLI group $(P=0.02)$. CLI and non-CLI groups also displayed differences in outcomes based on Kaplan-Meier survival analysis for MALE and re-interventions (Figure 1).

\section{Walking distance}

Pain-free walking distance and maximal walking distance improved only in the non-CLI group $(P=0.02$ and $P=0.01$, respectively), however, there were no differences between the groups ( $P=0.13$ and $P=0.11$, respectively; Table 2$)$.

\section{IMT}

\section{The relationship between clinical outcomes and baseline IMT}

The IMT value $>0.9 \mathrm{~mm}$ was found in 36 patients $(50.7 \%)$ from the overall group. The re-interventions occurred in 10 patients with IMT $>0.9 \mathrm{~mm}$ out of 13 found in the whole group (76.9\%), while considering MALEs there were 11 patients with IMT $>0.9 \mathrm{~mm}$ out of 16 found in the overall group (68.7\%). The relationship between baseline IMT and clinical outcomes showed that CLI patients with higher initial mean IMT (thicker than $0.9 \mathrm{~mm}$ ) value experienced the need for a larger number of re-interventions $(r=0.45$; $P=0.01)$ and larger number of MALEs $(r=0.45 ; P=0.01)$. 


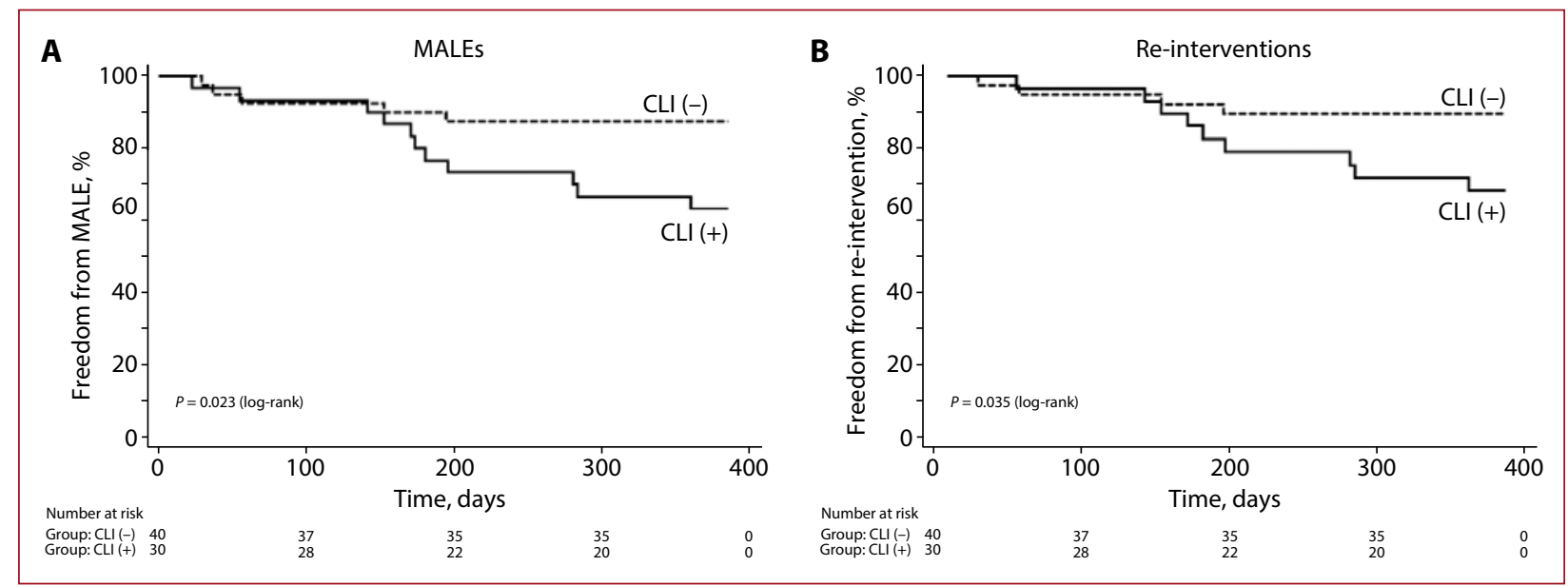

Figure 1. A. Kaplan-Meier survival curves according to critical limb ischemia (CLI) and non-CLI status for major adverse lower limb events. B. Kaplan-Meier survival curves according to CLI and non-CLI status for re-interventions

Abbreviations: MALE, major adverse limb events

Table 2. Changes of endothelial function parameters in critical limb ischemia (CLI) and non-CLI patients during the 6-month follow-up period following angioplasty

\begin{tabular}{|c|c|c|c|c|c|c|c|c|c|}
\hline \multirow[t]{2}{*}{ Variable } & \multicolumn{3}{|c|}{ CLI } & \multirow[t]{2}{*}{$P$-value } & \multicolumn{3}{|c|}{ Non-CLI } & \multirow[t]{2}{*}{$P$-value } & \multirow[t]{2}{*}{$P$-value ${ }^{a}$} \\
\hline & At baseline & 1 month & 6 months & & At baseline & 1 month & 6 months & & \\
\hline FMD & $3.0(1.7-4.7)$ & $5.2(3.3-6.4)$ & $2.9(1.9-5.3)$ & 0.16 & $4.4(2.1-6.6)$ & $3.9(2.6-6.5)$ & $2.4(1.3-4.4)$ & 0.08 & 0.26 \\
\hline PP & $66(56-76)$ & $63(56-80)$ & $72(56-80)$ & 0.88 & $71(69-75)$ & $66(58-78)$ & $62(53-70)$ & 0.4 & 0.74 \\
\hline $\mathrm{Al}$ & $100.5(88-115)$ & $92.5(82-107)$ & $98(83-105)$ & 0.65 & $94(85-110)$ & 94 (87-109) & $93.5(88-99.5)$ & 0.23 & 0.74 \\
\hline Central Al & $149(138-178)$ & $148(130-165)$ & $151(133-170)$ & 0.79 & $156(135-172)$ & $163(142-168)$ & $148(138-158.7)$ & 0.12 & 0.85 \\
\hline ED & $323(289-337)$ & $322(294-342)$ & $336(294-346)$ & 0.6 & $308(290-330)$ & $305(292-329)$ & $314.5(301.7-330)$ & 0.58 & 0.81 \\
\hline SEVR & $154(133-171)$ & $145(118-156)$ & $148(131-160)$ & 0.61 & $144.5(132-165.5)$ & $149(126-160)$ & $144.5(133-162.5)$ & 0.89 & 0.99 \\
\hline CAP & $17(13.5-26)$ & $18(14-23.5)$ & $18(12-27)$ & 0.91 & $17(13-27)$ & $20(13-23)$ & $15.5(12.2-21.5)$ & 0.25 & 0.89 \\
\hline CAP HR75 & $17(11.5-19.5)$ & $14.5(13.2-19.5)$ & $14(10-20.5)$ & 0.77 & $16(14-21)$ & $16(14-21)$ & $13(11-17.2)$ & 0.03 & 0.61 \\
\hline $\mathrm{SI}$ & $20(20-20)$ & $20(19-20)$ & $20(20-20)$ & 0.93 & $20(18-20)$ & $20(17-20)$ & $20(20-20)$ & 0.66 & 0.15 \\
\hline RI & $100(100-100)$ & $100(97-100)$ & $100(100-100)$ & 0.94 & $100(94-100)$ & $100(80.1-100)$ & $100(100-100)$ & 0.58 & 0.07 \\
\hline RHI & $1.46(1.19-1.81)$ & $1.37(1.08-1.62)$ & $1.44(1.16-1.98)$ & 0.66 & $1.59(1.22-2.26)$ & $1.68(1.32-2.22)^{*}$ & * $1.46(1.32-1.73)$ & 0.16 & 0.69 \\
\hline PFWD, m & $50(8.75-200)$ & $100(50-200)$ & $125(82.5-200)$ & 0.39 & $50(27.5-100)$ & $200(47.5-500)$ & $100(35-400)$ & 0.02 & 0.13 \\
\hline MWD, $m$ & $100(10-250)$ & $200(70-200)$ & $200(87.5-300)$ & 0.27 & $100(30-150)$ & $300(50-500)$ & $150(82.5-500)$ & 0.01 & 0.11 \\
\hline $\mathrm{SBP}, \mathrm{mm} \mathrm{Hg}$ & $143.8(18.2)$ & $145.6(16.3)$ & $149.4(18.6)$ & 0.56 & $150(14.8)$ & $146(19.3)$ & $142.2(16.7)$ & 0.18 & 0.35 \\
\hline $\mathrm{DBP}, \mathrm{mm} \mathrm{Hg}$ & $77.1(9.2)$ & $78.1(7.6)$ & $79.8(7.0)$ & 0.52 & $81.9(6.8)^{b}$ & $80.2(7.0)$ & $79.1(10.5)$ & 0.41 & 0.1 \\
\hline $\mathrm{IMT}, \mathrm{mm}$ & $0.94(0.3)$ & - & $0.91(0.26)$ & 0.055 & $0.97(0.29)$ & - & $0.94(0.26)$ & 0.23 & 0.88 \\
\hline
\end{tabular}

Data are expressed as mean (SD) and median (IQR).

aComparison between CLI and non-CLI with ANOVA Friedman's test for two groups with repeated measurements (2-way repeated measures ANOVA test).

$\mathrm{b} P<0.05$ when comparing particular indices at corresponding time points in the $\mathrm{CLI}$ and non-CLI groups.

Abbreviations: Al, coronary augmentation index; CAP, central augmentation pressure; CAP HR75, CAP normalized for the heart rate of 75 beats per minute; DBP, diastolic blood pressure; ED, ejection duration; FMD, flow mediated dilatation; IMT, intima-media thickness; MWD, maximal walking distance; PFWD, pain-free walking distance; PP, pulse pressure; RHI, reactive-hyperemia index; RI, reflection index; SBP, systolic blood pressure; SEVR, subendocardial viability ratio; SI, stiffness index

\section{Clinical outcomes}

\section{Changes in Rutherford scale and TBI after PTA in CLI and non-CLI patients}

Clinical outcomes assessed as the Rutherford grade revealed that during the follow-up, the Rutherford grade decreased significantly in the CLI group and did not change in the non-CLI (Figure 2). TBI did not change at the following time points in the $\mathrm{CLI}$ and non-CLI groups, and did not differ between either of the groups (Figure 2).

\section{Endothelial function}

\section{Changes in FMD after PTA in CLI and non-CLI patients}

The FMD measurements did not change during the following time points of the observational period in the CLI or non-CLI group ( $P=0.16$ and $P=0.08)$. FMD response to PTA did not differ between these groups (Table 2). Analyzing the measurements at given time points following PTA, we noted no significant differences (Figure 2). 
A

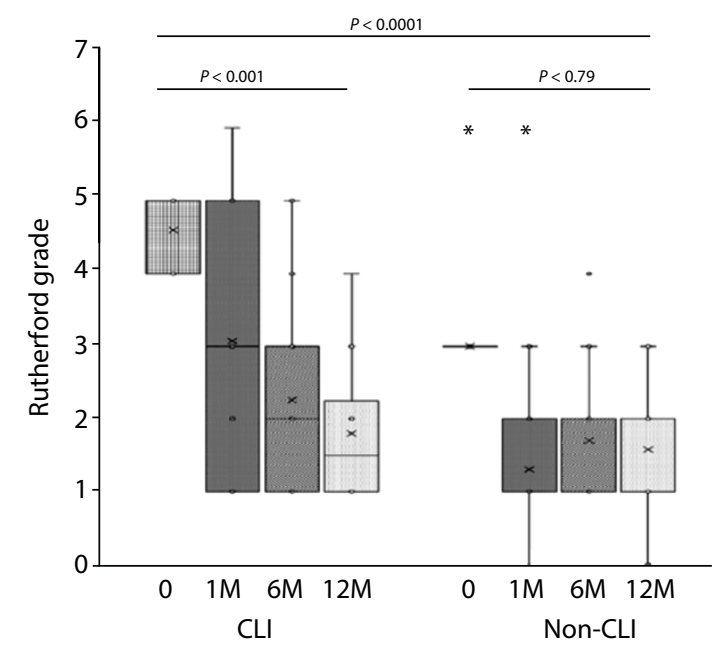

C

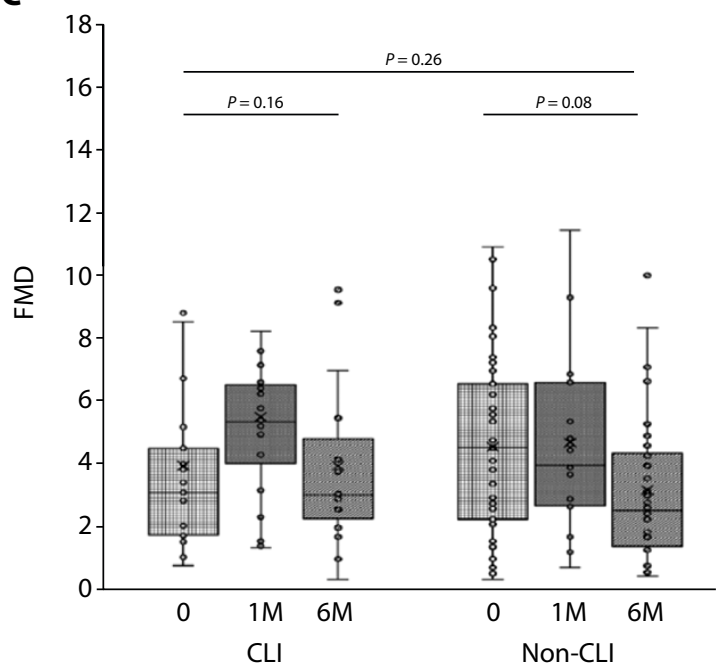

B

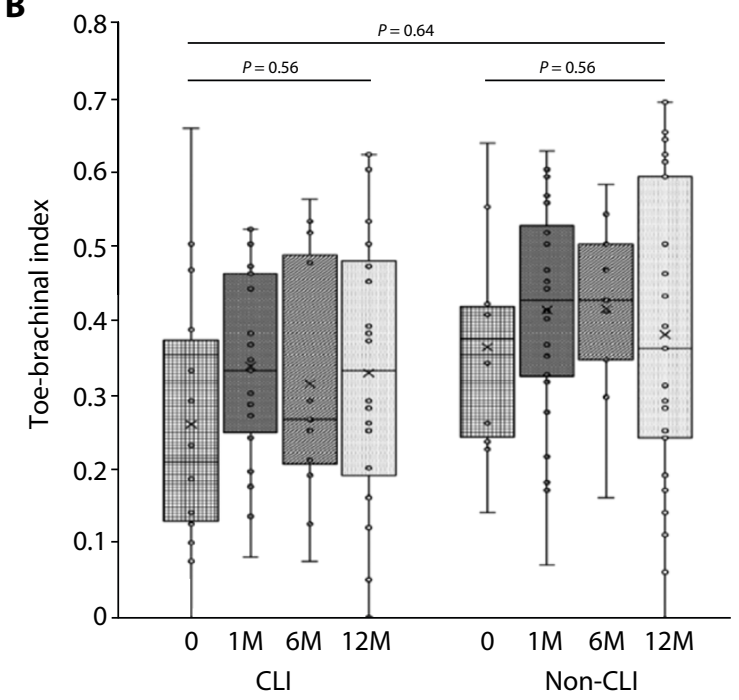

D

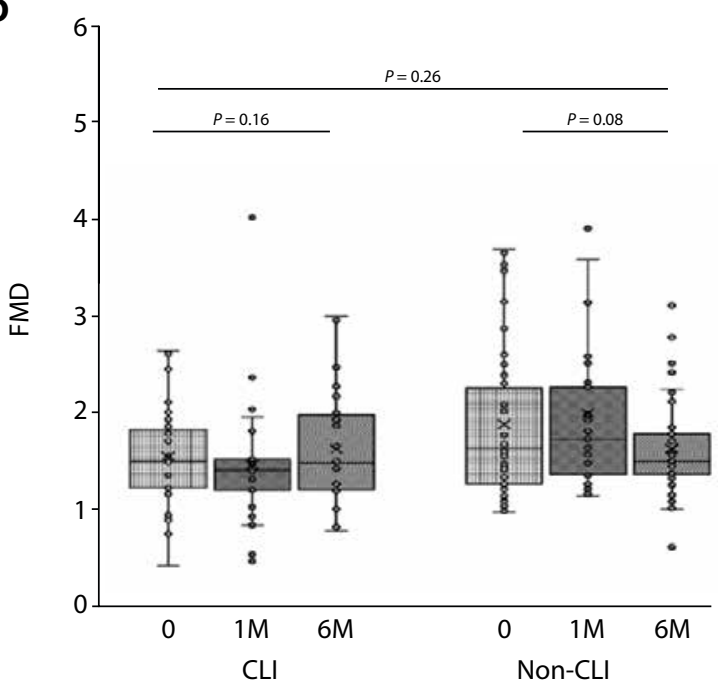

Figure 2. A. Rutherford grade in critical limb ischemia (CLI) and non-CLI patients at selected time points. B. Toe-brachial index in CLI and non-CLI patients at selected time points. C. Flow mediated dilatation in CLI and non-CLI patients at selected time points. D. Reactive-hyperemia index in CLI and non-CLI patients at selected time points. The horizontal line in the box indicates the median, the boxes indicate lower and upper quartiles, the cross in the box indicates the mean, the whiskers indicate standard deviation. M denotes month

\section{Changes in RHI after PTA in CLI and non-CLI patients}

Similarly to FMD, RHI did not change during the follow-up period in the $\mathrm{CLI}$ or non-CLI groups (Table 2). In contrast to FMD, calculating relative changes between measurements at given time points following PTA, changes in $\mathrm{RHI}(\triangle \mathrm{RHI})$ were negligible in the $\mathrm{CLI}$ and non-CLI groups (Table 2, 3 and Figure 2). However, $\triangle \mathrm{RHI} 1-6$ differed between the $\mathrm{CLI}$ and non-CLI groups $(P=0.01)$.

\section{Changes in PWA after PTA in CLI and non-CLI patients}

No significant changes were observed for PWA indices when considering the following time points in the $\mathrm{CLI}$ and non-CLI group, or comparing both groups (Table 2).

\section{DISCUSSION}

In the present study, we compared changes within endothelial function and clinical outcome after PTA in a group of 30 patients with $\mathrm{CLI}$ symptoms and a group of 40 patients with limb claudication. With regard to the clinical aspects of our study, we noted a significant increase in walking distance after PTA among patients with limb claudication as well as improved wound healing in the $\mathrm{CLI}$ patients. In the latter group, the Rutherford scale improved considerably $(P<0.001)$, and thus improvement remained longer, than in non-CLI patients.

We also used $\mathrm{ABI}$ and TBI to improve assessment regarding the clinical status of PAD patients. In CLI patients, we focused on TBI since it has been shown to present better accuracy in CLI diagnosis than ABI [7-9]. In this study, the 
Table 3. Comparison of relative changes in endothelial and clinical parameters between measurements at given time periods after percutaneous transluminal angioplasty in the critical limb ischemia (CLI) and non-CLI groups

\begin{tabular}{|c|c|c|c|c|}
\hline Variable & $\begin{array}{c}\text { CLI } \\
(n=30)\end{array}$ & $\begin{array}{l}\text { Non-CLI } \\
(n=40)\end{array}$ & $P$-value & $P$-value ${ }^{a}$ \\
\hline$\triangle \mathrm{FMD} 0-1$ & $1.5(-1.74-3.55)$ & $0.82(-2.06-2.95)$ & 0.75 & 0.91 \\
\hline$\triangle \mathrm{FMD} 0-6$ & $0(0-0.29)$ & $0(0-0.31)$ & 0.3 & 0.9 \\
\hline$\triangle F M D 1-6$ & $-5.11(-6.43--2.04)$ & $-4.18(-6.45--3.31)$ & 0.91 & 0.91 \\
\hline$\triangle \mathrm{RHI} 0-1$ & $-0.07(-0.31-0.17)$ & $0.1(-0.55-0.75)$ & 0.31 & 0.31 \\
\hline$\triangle \mathrm{RHI} 0-6$ & $0.18(-0.45-0.41)$ & $-0.18(-0.91-0.24)$ & 0.14 & 0.21 \\
\hline$\Delta \mathrm{RHI} 1-6$ & $0.08(-0.13-0.58)$ & $-0.15(-0.5-0.01)$ & 0.01 & 0.03 \\
\hline$\triangle A B I 0-6$ & $0.12(-0.17-0.23)$ & $0.09(-0.005-0.25)$ & 0.66 & 0.79 \\
\hline$\triangle \mathrm{ABI} 0-12$ & $-0.1(-0.31-0.12)$ & $0.005(-0.1-0.14)$ & 0.2 & 0.4 \\
\hline$\triangle A B I 1-6$ & $-0.07(-0.16-0.06)$ & $0.05(-0.07-0.14)$ & 0.03 & 0.12 \\
\hline$\Delta \mathrm{ABI} 1-12$ & $-0.11(-0.2-0.01)$ & $-0.04(-0.26-0.12)$ & 0.79 & 0.79 \\
\hline$\Delta \mathrm{TBI}$ 0-6 & $0.09(-0.08-0.16)$ & $0.005(-0.19-0.19)$ & 0.69 & 0.75 \\
\hline$\Delta \mathrm{TBI} 0-12$ & $-0.04(-0.24--0.003)$ & $0.02(-0.16-0.12)$ & 0.25 & 0.62 \\
\hline$\Delta \mathrm{TBI} 1-6$ & $0.01(-0.05-0.04)$ & $-0.04(-0.08-0.008)$ & 0.31 & 0.62 \\
\hline$\Delta \mathrm{TBI} 1-12$ & $-0.03(-0.13-0.09)$ & $-0.05(-0.19-0.11)$ & 0.75 & 0.75 \\
\hline$\Delta$ Rutherford 0-6 & $-3(-3.25--1.75)$ & $-2(-2--1)$ & $<0.001$ & $<0.007$ \\
\hline$\Delta$ Rutherford 0-12 & $-3(-4--2)$ & $-2(-2--1)$ & $<0.001$ & $<0.001$ \\
\hline$\Delta$ Rutherford 1-6 & $0(-3-0)$ & $0(0-1)$ & 0.004 & 0.004 \\
\hline$\Delta$ Rutherford 1-12 & $-1(-4-0)$ & $0(-1-1)$ & $<0.001$ & $<0.001$ \\
\hline$\triangle \mathrm{IMT}$ 0-6 & $-0.07(-0.42--0.002)$ & $-0.03(-0.17-0.06)$ & 0.07 & 0.07 \\
\hline
\end{tabular}

Data are expressed as median (IQR); $0-1$ indicates the difference between the first month following the procedure and baseline value; $0-6$ - the difference between the sixth month following the procedure and baseline value; $0-12$ - the difference between the twelfth month following the procedure and baseline value.

a $P$ - after adjustment by Benjamini-Hochberg procedure.

Abbreviations: $\mathrm{ABI}$, ankle-brachial index; $\mathrm{TBI}$, toe-brachial index; other: see Table 1 and 2

post-procedural increase in $\mathrm{ABI}$ and $\mathrm{TBI}$ was observed both in the CLI and non-CLI groups. However, based on the analyzed results, the TBI was the factor that improved predominantly in CLI patients. In contrast with non-CLI patients, the improvement in CLI group was observed till the end of the follow-up.

Another interesting finding of this study was achieved by the analysis of IMT. We noted that CLI patients with higher mean baseline IMT had significantly poorer clinical outcomes $(P=0.01$ for re-interventions and $P=0.03$ for major adverse events). Indeed, IMT has significant predictive value in assessing the risk of cardiovascular and cerebrovascular events [12, 17-20]. In several studies, a strong correlation has been shown between increased IMT and peripheral artery occlusive disease [21-23]. It is presumed that by reducing the IMT, we reduce vessel stiffness, which may directly translate into improved claudication distance. Improved blood supply to the arteries of the lower limbs (increased blood flow to the peripheral parts of the lower limbs), directly translates into a longer claudication distance and increased patient mobility (a kind of walking training), which has been shown to reduce arterial stiffness and improve endothelial function.

Endothelial function in $\mathrm{CLI}$ and non-CLI patients was measured on the basis of FMD and RHI measurements, which are well-established methods used in the assessment of endothelial dysfunction in patients with cardiovascular disease in conduit and resistance vessels, respectively [24]. In previous studies using FMD and $\mathrm{RHI}$, it has been shown that the FMD response and $\mathrm{RHI}$ score reflect endothelial status and, to some degree, NO-bioavailability [18]. The $\mathrm{RHI}$ correlates with the measurement of endothelial vasodilator function in the coronary arteries and with brachial FMD, although it has been suggested in other studies that FMD and RHI do not always correlate $[25,26]$. Nevertheless, both endothelial function tests significantly predicted cardiovascular events [27, 28].

In the present work, neither FMD nor RHI significantly improved during the follow-up in CLI or non-CLI patients. $\triangle F M D$ improved during the first month of observation in both groups, however, this improvement did not sustain as $\triangle F M D$ decreased between the first and sixth month following PTA. Similarly in RHI measurements, $\triangle \mathrm{RHI}$ displayed only transient improvement after the first month of PTA in CLI patients, and there were no significant differences between the first and sixth month in CLI and non-CLI group.

These results indicate that improvement of endothelial function after revascularization was transient and lasted only up to the first month following PTA. After that time, endothelial function deteriorated, even though patients displayed clinical benefits from revascularization.

Some previous reports confirmed the beneficial effects of peripheral artery revascularization on all-cause and 
cardiovascular mortality as well as improvement of global endothelial [29-32]. On the other hand, Budzyński et al. [33] reported that superficial femoral artery (SFA) stenting and classical pharmacotherapy did not significantly modify systemic endothelial function, as assessed by FMD in patients with adequately controlled individual risk factors of atherosclerosis. In contrast, among patients who did not reach treatment targets before SFA stenting, in those who underwent intensification of pharmacotherapy prior to the endovascular procedure, noticeable improvement in endothelial function markers (FMD, IMT) was observed within 3-6 months of observation.

The results of the present work suggest that neither PTA nor newly introduced pharmacological treatment (aspirin, clopidogrel, statin) had significant and sustained impact on post-PTA endothelial function. This may be due to several reasons. Firstly, we did not record compliance with the newly prescribed medications. We did not evaluate the level of control of patients' comorbidities, such as hypertension or diabetes. Furthermore, the follow-up period might have been too short, since most of the CLI patients required several months for the wounds to heal (up to 6 months). Based on the study, we may presume that a possible positive response of endothelial function to PTA and treatment was too weak, so intensification of pharmacological treatment would be needed to afford sustained improvement of endothelial function and more sustained benefits in PAD patients. Accordingly, endothelial-guided therapy may provide a tool to improve the efficacy of vascular pharmacotherapy that may be greater in non-CLI then CLI patients given the advanced disease in the latter group [34, 35].

\section{Study limitations}

The major study limitation of the present work was a relatively low number of patients included in the study. Furthermore, a modification of treatment (all patients were treated with statin, aspirin, and clopidogrel after PTA), improvement of patient compliance, modification of lifestyle (e.g. reduction in the number of cigarettes smoked, quitting smoking, or increasing physical activity) could also improve endothelial function after PTA, thus, it is difficult to interpret if endothelial function changes reported here were strictly related to endovascular procedure or could be the consequence of lifestyle changes, or medications.

\section{Article information}

Conflict of interest: None declared.

Open access: This article is available in open access under Creative Common Attribution-Non-Commercial-No Derivatives 4.0 International (CC BY-NC-ND 4.0) license, allowing to download articles and share them with others as long as they credit the authors and the publisher, but without permission to change them in any way or use them commercially. For commercial use, please contact the journal office at kardiologiapolska@ptkardio.pl.

How to cite: Kaczmarczyk P, Frołow M, Januszek R, et al. Endothelial function in patients with critical and non-critical limb ischemia undergoing endovascular treatment. Kardiol Pol. 2021; 79(7-8): 804-812, doi: 10.33963/KP.a2021.0010.

\section{REFERENCES}

1. Fowkes F, Rudan D, Rudan I, et al. Comparison of global estimates of prevalence and risk factors for peripheral artery disease in 2000 and 2010 : a systematic review and analysis. Lancet. 2013; 382(9901): 1329-1340, doi: 10.1016/s0140-6736(13)61249-0, indexed in Pubmed: 23915883.

2. Norgren L, Hiatt WR, Dormandy JA, et al. TASC II Working Group. Inter-society consensus for the management of peripheral arterial disease (TASC II). J Vasc Surg. 2007; 45(Suppl S): S5-S67, doi: 10.1016/j.jvs.2006.12.037, indexed in Pubmed: 17223489.

3. Conte MS, Pomposelli FB. Society for Vascular Surgery Practice guidelines for atherosclerotic occlusive disease of the lower extremities management of asymptomatic disease and claudication. Introduction. J Vasc Surg. 2015; 61(Suppl 3): 1S, doi: 10.1016/j.jvs.2014.12.006, indexed in Pubmed: 25721065.

4. Koutsiaris AG. Deep tissue near infrared second derivative spectrophotometry for the assessment of claudication in peripheral arterial disease. Clin Hemorheol Microcirc. 2017; 65(3): 275-284, doi: 10.3233/CH-16181, indexed in Pubmed: 27983543.

5. Romiti M, Albers M, Brochado-Neto FC, et al. Meta-analysis of infrapopliteal angioplasty for chronic critical limb ischemia. J Vasc Surg. 2008; 47(5): 975-981, doi: 10.1016/j.jvs.2008.01.005, indexed in Pubmed: 18372148.

6. Scali ST, Rzucidlo EM, Bjerke AA, et al. Long-term results of open and endovascular revascularization of superficial femoral artery occlusive disease. J Vasc Surg. 2011; 54(3): 714-721, doi: 10.1016/j.jvs.2011.03.216, indexed in Pubmed: 21620611.

7. Trocciola SM, Chaer R, Dayal R, et al. Comparison of results in endovascular interventions for infrainguinal lesions: claudication versus critical limb ischemia. Am Surg. 2005; 71(6): 474-479, discussion 479-480, doi: 10.1177/000313480507100605, indexed in Pubmed: 16044925.

8. Sachs T, Pomposelli F, Hamdan A, et al. Trends in the national outcomes and costs for claudication and limb threatening ischemia: angioplasty vs bypass graft. J Vasc Surg. 2011; 54(4): 1021-1031.e1, doi: 10.1016/j. jvs.2011.03.281, indexed in Pubmed: 21880457.

9. Widlansky ME, Gokce N, Keaney JF, et al. The clinical implications of endothelial dysfunction. J Am Coll Cardiol. 2003; 42(7): 1149-1160, doi: 10.1016/s0735-1097(03)00994-x, indexed in Pubmed: 14522472.

10. Kitta Y, Obata JE, Nakamura T, et al. Persistent impairment of endothelial vasomotor function has a negative impact on outcome in patients with coronary artery disease. J Am Coll Cardiol. 2009; 53(4): 323-330, doi: 10.1016/j.jacc.2008.08.074, indexed in Pubmed: 19161880.

11. Pawlaczyk K, Gabriel M, Urbanek T, et al. Changes in flow-mediated dilatation in patients with femoropopliteal occlusion receiving conservative and invasive treatment. Kardiol Pol. 2016; 74(8): 772-778, doi: 10.5603/KP.a2016.0027, indexed in Pubmed: 26965925.

12. Yeung AC, Vekshtein VI, Krantz DS, et al. The effect of atherosclerosis on the vasomotor response of coronary arteries to mental stress. N Engl J Med. 1991; 325(22): 1551-1556, doi: 10.1056/NEJM199111283252205, indexed in Pubmed: 1944439.

13. Greyling A, van Mil AC, Zock PL, et al. TIFN International Working Group on Flow Mediated Dilation. Adherence to guidelines strongly improves reproducibility of brachial artery flow-mediated dilation. Atherosclerosis. 2016; 248: 196-202, doi: 10.1016/j.atherosclerosis.2016.03.011, indexed in Pubmed: 27023841.

14. Zieliński B, Dróżdż A, Frołow M. Fully-automatic method for assessment of flow-mediated dilation. In: Chmielewski J, Datta A, Kozera R, Wojciechowski K. ed. Computer vision and graphics. Springer, Cham 2016: 439-450.

15. Hamburg NM, Keyes MJ, Larson MG, et al. Cross-sectional relations of digital vascular function to cardiovascular risk factors in the Framingham Heart Study. Circulation. 2008; 117(19): 2467-2474, doi: 10.1161/CIRCULATIONAHA.107.748574, indexed in Pubmed: 18458169.

16. Benjamini $Y$, Hochberg Y. Controlling the false discovery rate: a practical and powerful approach to multiple testing. J R Stat Soc Series B Stat Methodol. 2018; 57(1): 289-300, doi: 10.1111/j.2517-6161.1995. tb02031.x.

17. Davis PH, Dawson JD, Riley WA, et al. Carotid intimal-medial thickness is related to cardiovascular risk factors measured from childhood through middle age: The Muscatine Study. Circulation. 2001; 104(23): 2815-2819, doi: 10.1161/hc4601.099486, indexed in Pubmed: 11733400. 
18. Nohria A, Gerhard-Herman M, Creager MA, et al. Role of nitric oxide in the regulation of digital pulse volume amplitude in humans. J Appl Physiol (1985). 2006; 101(2): 545-548, doi: 10.1152/japplphysiol.01285.2005, indexed in Pubmed: 16614356.

19. Miszalski-Jamka T, Lichołai S, Karwat K, et al. Computed tomography characteristics of coronary artery atherosclerosis in subjects with lower extremity peripheral artery disease and no cardiac symptoms. Pol Arch Med Wewn. 2013; 123(12): 657-663, doi: 10.20452/pamw.2005, indexed in Pubmed: 24185038.

20. Gacoń J,Przewłocki T, Podolec J, et al. Prospective study on the prognostic value of repeated carotid intima-media thickness assessment in patients with coronary and extra coronary steno-occlusive arterial disease. Pol Arch Intern Med. 2019; 129(1): 12-21, doi: 10.20452/pamw.4407, indexed in Pubmed: 30600311.

21. Bots ML, Hofman A, Grobbee DE. Common carotid intima-media thickness and lower extremity arterial atherosclerosis. the Rotterdam study. Arterioscler Thromb. 1994; 14(12): 1885-1891, doi: 10.1161/01.atv.14.12.1885, indexed in Pubmed: 7981175.

22. Bots $M$, de Jong PT, Hofman $A$, et al. Left, right, near or far wall common carotid intima-media thickness measurements: associations with cardiovascular disease and lower extremity arterial atherosclerosis. J Clin Epidemiol. 1997; 50(7): 801-807, doi: 10.1016/s0895-4356(97)00059-0, indexed in Pubmed: 9253391.

23. Allan PL, Mowbray PI, Lee AJ, et al. Relationship between carotid intima-media thickness and symptomatic and asymptomatic peripheral arterial disease. The Edinburgh artery study. Stroke. 1997; 28(2):348-353, doi: 10.1161/01.str.28.2.348, indexed in Pubmed: 9040688.

24. Flammer AJ, Anderson T, Celermajer DS, et al. The assessment of endothelial function: from research into clinical practice. Circulation. 2012; 126(6): 753-767, doi: 10.1161/CIRCULATIONAHA.112.093245, indexed in Pubmed: 22869857.

25. Bonetti PO, Pumper GM, Higano ST, et al. Noninvasive identification of patients with early coronary atherosclerosis by assessment of digital reactive hyperemia. J Am Coll Cardiol. 2004;44(11):2137-2141, doi: 10.1016/j. jacc.2004.08.062, indexed in Pubmed: 15582310.

26. Kuvin J, Patel A, Sliney K, et al. Assessment of peripheral vascular endothelial function with finger arterial pulse wave amplitude. Am Heart J. 2003; 146(1): 168-174, doi: 10.1016/s0002-8703(03)00094-2, indexed in Pubmed: 12851627.
27. Matsuzawa Y, Kwon TG, Lennon RJ, et al. Prognostic value of flow-mediated vasodilation in brachial artery and fingertip artery for cardiovascular events: a systematic review and meta-analysis. J Am Heart Assoc. 2015; 4(11): e002270, doi: 10.1161/JAHA.115.002270, indexed in Pubmed: 26567372.

28. Hirata Y, Nagata D, Suzuki E, et al. Diagnosis and treatment of endothelial dysfunction in cardiovascular disease. Int Heart J. 2010; 51(1): 1-6, doi: 10.1536/ihj.51.1, indexed in Pubmed: 20145343.

29. Giugliano G, Di Serafino L, Perrino C, et al. Effects of successful percutaneous lower extremity revascularization on cardiovascular outcome in patients with peripheral arterial disease. Int J Cardiol. 2013; 167(6): 25662571, doi: 10.1016/j.ijcard.2012.06.055, indexed in Pubmed: 22790191.

30. Diehm C, Allenberg JR, Pittrow D, et al. German Epidemiological Trial on Ankle Brachial Index Study Group. Mortality and vascular morbidity in older adults with asymptomatic versus symptomatic peripheral artery disease. Circulation. 2009; 120(21): 2053-2061, doi: 10.1161/CIRCULATIONAHA.109.865600, indexed in Pubmed: 19901192.

31. Unal $\mathrm{O}$, Karatepe $\mathrm{O}$, Ugurlucan $\mathrm{M}$, et al. Effects of lower extremity revascularization on the endothelial functions measured with noninvasive brachial artery flow-mediated dilatation. Ann Vasc Surg. 2011; 25(7): 969-974, doi: 10.1016/j.avsg.2011.02.013, indexed in Pubmed: 21530156.

32. Hamburg NM, Palmisano J, Larson MG, et al. Relation of brachial and digital measures of vascular function in the community: the Framingham heart study. Hypertension. 2011; 57(3): 390-396, doi: 10.1161/HYPERTENSIONAHA.110.160812, indexed in Pubmed: 21263120.

33. Budzyński J, Wiśniewska J, Wasielewski M, et al. The effect of superficial femoral artery stenting and some atherosclerosis risk factors on changes in selected global endothelial function tests in patients with chronic lower limb ischemia. A pilot study. Postep Kardiol Inter. 2012; 8(3): 205-215, doi: 10.5114/pwki.2012.30400.

34. Walczak M, Suraj J, Kus K, et al. Towards a comprehensive endothelial biomarkers profiling and endothelium-guided pharmacotherapy. Pharmacol Rep. 2015; 67(4): 771-777, doi: 10.1016/j.pharep.2015.06.008, indexed in Pubmed: 26321280.

35. Frolow M, Drozdz A, Kowalewska A, et al. Comprehensive assessment of vascular health in patients; towards endothelium-guided therapy. Pharmacol Rep. 2015;67(4): 786-792, doi: 10.1016/j.pharep.2015.05.010, indexed in Pubmed: 26321282. 